Research Article

\title{
High-Accuracy and Low-Cost Attitude Measurement Unit of the CubeSat
}

\author{
Haining Ma, ${ }^{1}$ Zhengliang Lu $\mathbb{D},{ }^{1}$ Xiang Zhang, ${ }^{1}$ Wenhe Liao, ${ }^{1}$ and Klaus Briess ${ }^{2}$ \\ ${ }^{1}$ Micro/Nano Satellite Research Center, School of Mechanical Engineering, Nanjing University of Science and Technology, \\ Nanjing 210094, China \\ ${ }^{2}$ Chair of Space Technology, Institute of Aeronautics and Astronautics, Technical University of Berlin, Berlin 10587, Germany
}

Correspondence should be addressed to Zhengliang Lu; 112010115@njust.edu.cn

Received 24 January 2020; Revised 2 July 2020; Accepted 31 July 2020; Published 28 August 2020

Academic Editor: Seid H. Pourtakdoust

Copyright (C) 2020 Haining Ma et al. This is an open access article distributed under the Creative Commons Attribution License, which permits unrestricted use, distribution, and reproduction in any medium, provided the original work is properly cited.

\begin{abstract}
This paper proposes high-accuracy and reliable attitude measurement methods exclusive for CubeSat with restrictions of low cost, limited space, and low power consumption. The attitude measurement unit is equipped with Commercial Off-The-Shelf (COTS) components including Micro-Electro-Mechanical System (MEMS) gyro and two simultaneously operating star trackers (STR) to enhance the measurement accuracy. The Multiplicative Extended Kalman Filter (MEKF) is used to estimate the attitude of CubeSat, and four kinds of attitude estimation layouts are put forward according to the idea of weighted average of two quaternions from two STR and different architectures of information fusion. Using the proposed methods, the attitude measurement unit can continuously provide accurate and reliable attitude knowledge for attitude control unit when the CubeSat is running in orbit. Numerical simulation is performed to verify the effectiveness of the proposed methods, and it offers a reference for CubeSat developers from the perspective of engineering application.
\end{abstract}

\section{Introduction}

CubeSat has become an interesting innovation in the space frontier due to advantages such as low cost, good flexibility, short development period, and high functional density [1-3]. The CubeSats used for imaging and videoing are mainstream among those that have been launched [4]. High-accuracy measurement and control are key to achieving the above tasks.

Gyro and STR are widely used in satellite with a high demand of attitude control accuracy. The small satellite Flying Laptop adopts one STR and four fiber-optic gyros (FOG) to accomplish inertial attitude measurement when the satellite is working in earth observation mode [5]; a novel STR named NSTT with high-accuracy attitude measurement capacity $\left(<4^{\prime \prime}\right)$ and high acquisition rate $\left(>3^{\circ} / \mathrm{s}\right)$ is utilized in Super Low Altitude Test Satellite (SLATS) as a technology demonstration payload [6]; the nanosatellite TechnoSat uses one FOG to integrate angular rate to generate attitude knowledge when the satellite is working in attitude maneuver mode [7]; there are two STR vertical to each other in the small sat- ellite Bispectral InfraRed Optical System (BIROS), one of them works as cold backup [8]. The above FOG and STR are mainly applied in nanosatellites or larger satellite platforms, although they have high enough measurement accuracy; however, they are not suitable for CubaSat platforms because of large size and high power consumption. For example, the size and power consumption of the STR used in Flying Laptop are $10 \times 10 \times 10 \mathrm{~cm}^{3}$ and $7.8 \mathrm{~W}$, respectively, while the size and maximum power supply of our $2 \mathrm{U}$ CubeSat are $20 \times 10 \times 10 \mathrm{~cm}^{3}$ and $4.6 \mathrm{~W}[9,10]$.

In recent years, many scholars have carried out studies on the attitude measurement of satellites with gyro and STR integrated. Ref. [11] proposes Moving Horizon Filter (MHF) to estimate the attitude and gyro calibration parameters by jointly using one STR and one gyro and draws a conclusion that the proposed method results in an increased accuracy on nonlinear systems with respect to Extended Kalman Filter (EKF); in Ref. [12], attitude fusion data obtained by Complementary Filter (CF) according to different noise frequency characteristics of one gyro and one STR is introduced as the observed values of the Unscented Kalman Filter 
(UKF) system to the process of measurement update, finally improving the accuracy of attitude estimation compared with only UKF used; in Ref. [13], Cubature Kalman Filter (CKF) is utilized to estimate satellite attitude aiming at the satellite star sensor/gyro attitude measurement system; the results indicate that CKF is better than EKF and UKF in accuracy and stability when dealing with strongly nonlinear attitude kinematics and dynamics equations; Ref. [14] proposes Robust Adaptive Unscented Kalman Filter (RAUKF) to achieve precise spacecraft attitude estimation in the presence of measurement faults by using one STR and one gyro and proves that the proposed algorithm outperforms the standard EKF and UKF under measurement faults through error covariance adaptation. The above literature mainly focuses on the research of attitude measurement algorithms, although the results show that all the proposed algorithms perform better than EKF in theory; however, they cannot perform as well as we expect in practical application. Firstly, the kinematics equation of CubeSat is not so strongly nonlinear, so filters applied to strongly nonlinear systems such as UKF, CKF, and their variants cannot provide more accurate attitude knowledge than EKF actually in this case. Secondly, UKF, $\mathrm{CKF}$, and their variants involve complicated mathematical calculation such as Cholesky and QR decomposition [15]; although we can invoke relevant functions in MATLAB directly just for numerical simulation, we have to rewrite the complex codes in other programming languages suitable for running in an on-board computer of CubeSat; moreover, a large amount of loop iteration and complicated function calculation in UKF, CKF, and their variants lead to more execution time than EKF; for example, the execution time of UKF is almost twice as much as EKF under the same simulation condition according to our comparison, which greatly increases computation burden of an on-board computer of CubeSat with limited capacity.

Physically, the rotation angle about the line-of-sight axis of STR is less accurate than that about the other two axes; hence, the total attitude measurement accuracy is influenced when only one STR is used [16]. Therefore, some scholars have carried out research on attitude measurement by using multiple STR. Singular Value Decomposition (SVD) and Quaternion Estimator (QUEST) algorithms are proposed in Ref. [17] to estimate the attitude of satellite based on stellar vectors from multiple (usually more than two) simultaneously operating STR; Ref. [18] reviews a kind of gyroless attitude measurement method which utilizes the TRIAD algorithm to estimate the attitude angles of satellite based on stellar vectors from two simultaneously operating STR then calculates the angular rate of a satellite by a first-order difference; Federated Extended Kalman Filter (FEKF) and Federated Unscented Kalman Filter (FUKF) are, respectively, proposed in Ref. [19] and Ref. [20]; the former contains three independent local filters using measurements from one gyro and three simultaneously operating STR and one master filter while the latter contains two independent local filters using measurements from one gyro and two simultaneously operating STR and one master filter, and their respective estimated results are fused in the master filter to finally generate the attitude knowledge of satellite. The above
SVD, QUEST, and TRIAD belong to single-frame methods; they do not deal with measurement errors of attitude sensors and use any knowledge about kinematics and dynamics of satellites compared with Kalman filter-based methods such as EKF, UKF, and CKF; hence, the Kalman filter-based methods generally provide more accurate estimates than the single-frame methods [21, 22]. For the large satellite equipped with high-accuracy attitude sensors, these algorithms can provide sufficiently accurate attitude knowledge; however, for the CubeSat equipped with relatively coarse attitude sensors, they are not good choices actually.

Inspired by the idea of federated filters proposed in Ref. [19] and Ref. [20] and a weighted average of two quaternions proposed in Ref. [23], we propose a novel attitude measurement method by using one gyro and two simultaneously operating STR in this paper, as part of the development task of the CubeSat NJUST-2. On the hardware level, we adopt COTS components with the properties of small size, low power dissipation, and low cost as attitude sensors, which include one MEMS gyro and two simultaneously operating STR; on the algorithm level, we develop four kinds of attitude estimation layouts based on EKF according to the architecture of information fusion; they are called centralized layout I, centralized layout II, centralized layout III, and decentralized layout.

The paper is organized as follows. Attitude kinematics of CubeSat and attitude sensor modeling are described in Section 2. Four kinds of attitude estimation layouts and attitude estimation processes based on MEKF are given in Section 3. Numerical simulation, comparison, and analysis are conducted in Section 4. Finally, conclusions are presented in Section 5 .

\section{Attitude Kinematics and Sensor Modeling}

2.1. Quaternion Kinematics. Quaternion is used to describe the attitude of CubeSat in our study due to its nonsingularity. The quaternion is defined as

$$
\mathbf{q}=\left[q_{0} \mathbf{q}_{v}^{T}\right]^{T}
$$

where $q_{0}$ is the scalar part; $\mathbf{q}_{v}$ is the vector part, $\mathbf{q}_{v}=$ $\left[\begin{array}{lll}q_{1} & q_{2} & q_{3}\end{array}\right]^{T}$.

The kinematic differential equation represented by quaternion is described as [14]

$$
\dot{\mathbf{q}}=\frac{1}{2} \Omega(\omega) \mathbf{q}
$$

where $\boldsymbol{\omega}$ is the angular rate vector; $\boldsymbol{\Omega}(\boldsymbol{\omega})$ is represented as [14]

$$
\boldsymbol{\Omega}(\boldsymbol{\omega})=\left[\begin{array}{cccc}
0 & -\omega_{x} & -\omega_{y} & -\omega_{z} \\
\omega_{x} & 0 & \omega_{z} & -\omega_{y} \\
\omega_{y} & -\omega_{z} & 0 & \omega_{x} \\
\omega_{z} & \omega_{y} & -\omega_{x} & 0
\end{array}\right]
$$


TABLE 1: PST3 pico STR performance.

\begin{tabular}{lc}
\hline Dimension & $3.3 \times 3.3 \times 3.7 \mathrm{~cm}^{3}$ \\
Field of view (FOV) & $15^{\circ} \times 12^{\circ}$ (effective) \\
Measurement accuracy & $<10^{\prime \prime}(3 \sigma$, pointing) \\
Stray light suppression angle & $>35^{\circ}$ \\
Power consumption & $<0.6 \mathrm{~W}$ \\
Output format & Quaternion \\
\hline
\end{tabular}

2.2. Gyro Modeling. The gyro body axes are aligned with principal axes of inertia of the CubeSat body; it can measure the angular rate of the CubeSat body frame relative to the geocentric inertial frame. Considering the presence of bias, measurement noise, and random drift, the discrete mathematical model of the gyro is built as [24]

$$
\begin{aligned}
\tilde{\boldsymbol{\omega}}_{b i} & =\overline{\boldsymbol{\omega}}_{b i}+\frac{1}{2}\left(\overline{\mathbf{b}}_{k}+\overline{\mathbf{b}}_{k-1}\right)+\mathbf{W}_{\omega}, \\
\overline{\mathbf{b}}_{k+1} & =\overline{\mathbf{b}}_{k}+\mathbf{W}_{b},
\end{aligned}
$$

where $\tilde{\boldsymbol{\omega}}_{b i}$ and $\overline{\boldsymbol{\omega}}_{b i}$ are the measured and true angular rate of the CubeSat body frame relative to the geocentric inertial frame, respectively; $\overline{\mathbf{b}}_{k+1}, \overline{\mathbf{b}}_{k}$, and $\overline{\mathbf{b}}_{k-1}$ are the true biases at time $k+1, k$, and $k-1$, respectively; $\mathbf{W}_{\omega}$ is the measurement noise, denoted as

$$
\mathbf{W}_{\omega}=\left(\frac{N^{2}}{T_{s}}+\frac{1}{12} K^{2} T_{s}\right)^{1 / 2} \boldsymbol{\eta},
$$

where $N$ and $K$ are the angle random walk and rate random walk, respectively; $T_{s}$ is the sampling period; $\boldsymbol{\eta}$ is the independent Gaussian white noise, $\boldsymbol{\eta} \sim N(0,1)$.

$\mathbf{W}_{b}$ is the bias noise, denoted as

$$
\mathbf{W}_{b}=K T_{s}^{1 / 2} \boldsymbol{\eta} .
$$

\subsection{STR Modeling}

2.3.1. Mounting and Layout. Two pico STR titled PST3 manufactured by TY-Space Technology are used in the attitude measurement unit of NJUST-2; its parameters are listed in Table 1.

In general, two STR are mounted at a tilted angle equal to $90^{\circ}$ for the highest measurement accuracy and complete observability [25]. Figure 1 shows the on-orbit flight model of the CubeSat built in Systems Tool Kit (STK). The CubeSat will run in a sunsynchronous orbit with an inclination of $97.5^{\circ}$; A1, B1, B2, and $\mathrm{C} 1$ are the primary surfaces illuminated by sunlight; $\mathrm{C} 2$ is the secondary surface illuminated by sunlight; A2 is the surface illuminated by reflected light form atmosphere.

Therefore, we consider mounting the two STR on C2 at a tilted angle equal to $90^{\circ}$ for protecting them from stray light disturbance, as Figure 2 shows.

To verify the effectiveness of the above mounting method, relevant simulation and analysis are carried out by STK. Since the two STR are not exposed to sunlight during the eclipse area, we only consider the sunlight area. Orbit

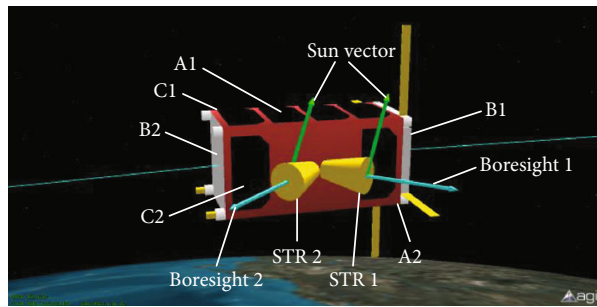

Figure 1: On-orbit flight model of CubeSat in STK.

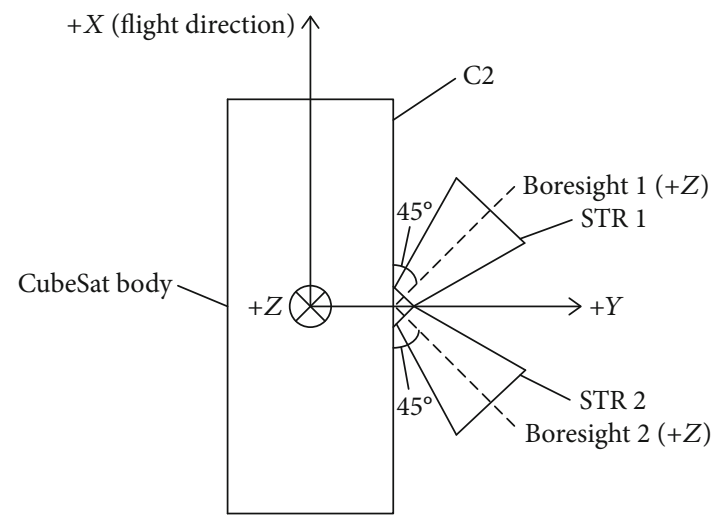

Figure 2: Mounting diagram of two STR.

parameters in our study are from Two-Line Element (TLE) sets of our last CubeSat NJUST-1 whose orbit is similar to NJUST-2, published by North American Aerospace Defense Command (NORAD). The first available TLE is as follows:

(1) $143156 \mathrm{U} 18008 \mathrm{~B} 18039.44439993 .0000018200000$ 0 15203-4 09998

(2) $2 \quad 43156 \quad 97.5404 \quad 116.0398 \quad 0014251 \quad 219.2554$ 201.516915 .097648443059

The initial orbit epoch is 8 Feb 2018 10:39:56.154 Coordinated Universal Time Greenwich (UTCG). Figure 3 illustrates the angles between sun vector and orbit plane titled beta angle within one year from the above date.

As Figure 3 shows, the beta angle is minimal at noon on June 1st. The angle between boresight 1 and sun vector titled $\alpha 1$ and the angle between boresight 2 and sun vector titled $\alpha 2$ are also minimal at that time, which means sunlight has the greatest influence on the two STR. Therefore, we only need to consider the above worst condition. Selecting 1 Jun 2018 12:10:56.997 UTCG 1 Jun 2018 13:11:20.285 UTCG as 


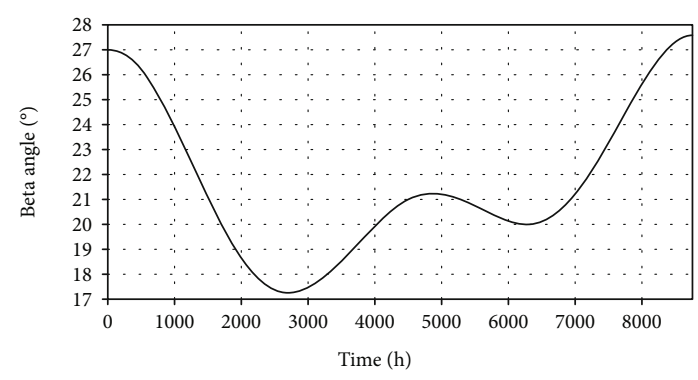

FIGURE 3: Beta angles within one year.

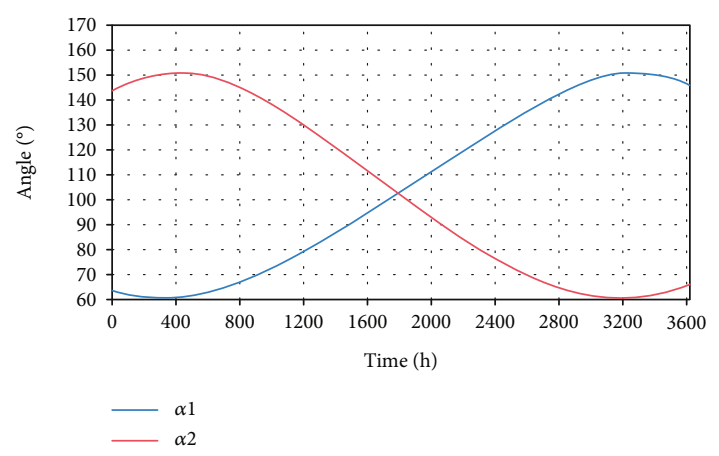

FIgURE 4: Angles between STR boresight and sun vector.

simulation duration, we can obtain $\alpha 1$ and $\alpha 2$ within the above period, as Figure 4 shows.

As Figure 3 shows, the minimums of $\alpha 1$ and $\alpha 2$ are both larger than the stray light suppression angle of the STR, which means that the mounting method of the two STR can effectively protect them from stray light disturbance during the CubeSat's lifetime.

2.3.2. Mathematical Model. The STR can measure attitude quaternion of the geocentric inertial frame relative to the STR body frame. Considering that the STR body axes are not aligned with principal axes of inertia of the CubeSat body and the presence of measurement noise, the discrete mathematical model of STR is built as

$$
\tilde{\mathbf{q}}_{b i}=\overline{\mathbf{q}}_{b i} \otimes \Delta \tilde{\mathbf{q}}_{b i},
$$

where $\tilde{\mathbf{q}}_{b i}$ and $\overline{\mathbf{q}}_{b i}$ are the measured and true attitude quaternion of the geocentric inertial frame relative to the CubeSat body frame, respectively; $\Delta \tilde{\mathbf{q}}_{b i}$ is the converted measurement noise, denoted as

$$
\begin{aligned}
\Delta \tilde{\mathbf{q}}_{b i} & =\overline{\mathbf{q}}_{b i}{ }^{-1} \otimes \tilde{\mathbf{q}}_{b i}=\left(\overline{\mathbf{q}}_{s i} \otimes \mathbf{q}_{b s}\right)^{-1} \otimes\left(\tilde{\mathbf{q}}_{s i} \otimes \mathbf{q}_{b s}\right) \\
& =\mathbf{q}_{b s}{ }^{-1} \otimes\left(\left(\overline{\mathbf{q}}_{s i}{ }^{-1} \otimes \tilde{\mathbf{q}}_{s i}\right) \otimes \mathbf{q}_{b s}\right)=\mathbf{q}_{b s}{ }^{-1} \otimes\left(\Delta \tilde{\mathbf{q}}_{s i} \otimes \mathbf{q}_{b s}\right),
\end{aligned}
$$

where $\tilde{\mathbf{q}}_{s i}$ and $\overline{\mathbf{q}}_{s i}$ are the measured and true attitude quaternion of the geocentric inertial frame relative to the STR body frame, respectively; $\mathbf{q}_{b s}$ is the attitude quaternion of the STR body frame relative to the CubeSat body frame, denoted as

$$
\begin{aligned}
& q_{b s 0}=\cos \frac{\theta_{b s}}{2} \cos \frac{\varphi_{b s}}{2} \cos \frac{\psi_{b s}}{2}-\sin \frac{\theta_{b s}}{2} \sin \frac{\varphi_{b s}}{2} \sin \frac{\psi_{b s}}{2} \\
& q_{b s 1}=\cos \frac{\theta_{b s}}{2} \sin \frac{\varphi_{b s}}{2} \cos \frac{\psi_{b s}}{2}-\sin \frac{\theta_{b s}}{2} \cos \frac{\varphi_{b s}}{2} \sin \frac{\psi_{b s}}{2}, \\
& q_{b s 2}=\sin \frac{\theta_{b s}}{2} \cos \frac{\varphi_{b s}}{2} \cos \frac{\psi_{b s}}{2}+\cos \frac{\theta_{b s}}{2} \sin \frac{\varphi_{b s}}{2} \sin \frac{\psi_{b s}}{2}, \\
& q_{b s 3}=\sin \frac{\theta_{b s}}{2} \sin \frac{\varphi_{b s}}{2} \cos \frac{\psi_{b s}}{2}+\cos \frac{\theta_{b s}}{2} \cos \frac{\varphi_{b s}}{2} \sin \frac{\psi_{b s}}{2}
\end{aligned}
$$

where $\psi_{b s}, \varphi_{b s}$, and $\theta_{b s}$ are the Euler angles of the STR body frame relative to the CubeSat body frame.

$\Delta \tilde{\mathbf{q}}_{s i}$ is the original measurement noise, under the condition of small angle; it can be denoted as

$$
\Delta \tilde{\mathbf{q}}_{s i}=\left[\sqrt{1-\left(\frac{\Delta r}{2} \boldsymbol{\eta}+\frac{\Delta p}{2} \boldsymbol{\eta}+\frac{\Delta p}{2} \boldsymbol{\eta}\right)^{2}} \frac{\Delta r}{2} \boldsymbol{\eta} \quad \frac{\Delta p}{2} \boldsymbol{\eta} \frac{\Delta p}{2} \boldsymbol{\eta}\right]^{T},
$$

where $\Delta r$ and $\Delta p$ are the measurement errors in the directions of rolling and pointing, respectively.

The measurement residual is denoted as

$$
\begin{aligned}
\delta \tilde{\mathbf{q}}_{b i} & =\mathbf{q} \wedge_{b i}{ }^{-1} \otimes \tilde{\mathbf{q}}_{b i}=\mathbf{q} \wedge_{b i}{ }^{-1} \otimes\left(\overline{\mathbf{q}}_{b i} \otimes \Delta \tilde{\mathbf{q}}_{b i}\right) \\
& =\left(\mathbf{q}_{o i} \otimes \mathbf{q} \wedge_{b o}\right)^{-1} \otimes\left(\mathbf{q}_{o i} \otimes \overline{\mathbf{q}}_{b o}\right) \otimes \Delta \tilde{\mathbf{q}}_{b i} \\
& =\left(\left(\mathbf{q} \wedge_{b o}{ }^{-1} \otimes \mathbf{q}_{o i}{ }^{-1}\right) \otimes \mathbf{q}_{o i}\right) \otimes \overline{\mathbf{q}}_{b o} \otimes \Delta \tilde{\mathbf{q}}_{b i} \\
& =\mathbf{q} \wedge_{b o}{ }^{-1} \otimes \overline{\mathbf{q}}_{b o} \otimes \Delta \tilde{\mathbf{q}}_{b i}=\Delta \widehat{\mathbf{q}}_{b o} \otimes \Delta \tilde{\mathbf{q}}_{b i},
\end{aligned}
$$

where $\widehat{\mathbf{q}}_{b i}$ is the estimated attitude quaternion of the geocentric inertial frame relative to the CubeSat body frame; $\mathbf{q}_{o i}$ is the attitude quaternion of the geocentric inertial frame relative to the orbit frame; $\widehat{\mathbf{q}}_{b o}$ and $\overline{\mathbf{q}}_{b o}$ are the estimated and true attitude quaternion of the orbit frame relative to the CubeSat body frame, respectively; $\Delta \widehat{\mathbf{q}}_{b o}$ is the estimation error.

Extracting the vector part of $\delta \tilde{\mathbf{q}}_{b i}$, the linear equation of measurement residual can be denoted as

$$
\delta \tilde{\mathbf{q}}_{b i_{-} v}=\Delta \widehat{\mathbf{q}}_{b o_{-} v}+\Delta \tilde{\mathbf{q}}_{b i_{-} v},
$$

where $\delta \tilde{\mathbf{q}}_{b i_{-} v}, \Delta \widehat{\mathbf{q}}_{b o_{-} v}$, and $\Delta \tilde{\mathbf{q}}_{b i_{-} v}$ are the vector part of $\delta \tilde{\mathbf{q}}_{b i}$, $\Delta \widehat{\mathbf{q}}_{b o}$, and $\Delta \tilde{\mathbf{q}}_{b i}$, respectively.

\section{Attitude Estimation Algorithm}

3.1. Attitude Estimation Layout. Due to the nonadditive characteristic of the quaternion, the common linear weighted average method is unable to directly calculate the average of multiple quaternions because it has the feature of nonuniqueness and cannot guarantee the normalization constraint of the quaternion. Aiming at the above problem, 


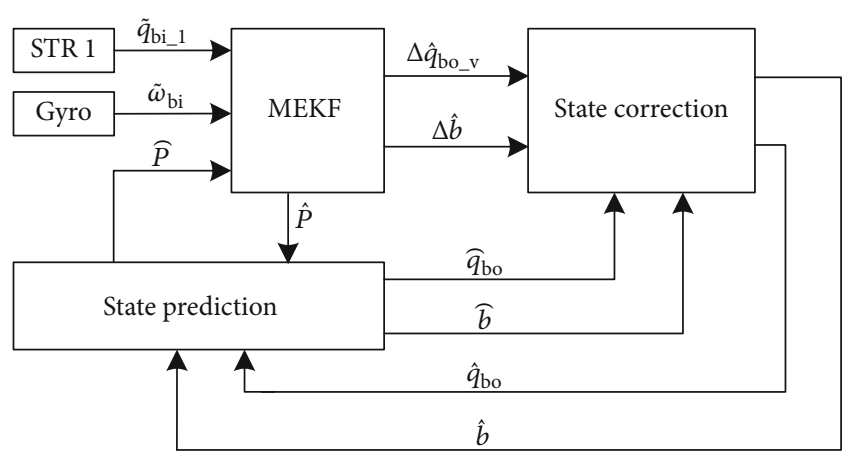

Figure 5: The centralized layout I.

Ref. [23] proposes an effective nonlinear weighted average method to calculate the average of two quaternions as follows:

$\mathbf{q}_{w}=\sqrt{\frac{w_{1}\left(w_{1}-w_{2}+z\right)}{z\left(w_{1}+w_{2}+z\right)}} \mathbf{q}_{1}+\operatorname{sign}\left(\mathbf{q}_{1}{ }^{T} \mathbf{q}_{2}\right) \sqrt{\frac{w_{2}\left(w_{2}-w_{1}+z\right)}{z\left(w_{1}+w_{2}+z\right)}} \mathbf{q}_{2}$,

where $z=\sqrt{\left(w_{1}-w_{2}\right)^{2}+4 w_{1} w_{2}\left(\mathbf{q}_{1}{ }^{T} \mathbf{q}_{2}\right)^{2}} ; \mathbf{q}_{1}$ and $\mathbf{q}_{2}$ are the two quaternions probably from two STR, Particle Filter (PF), multiple-model adaptive estimation, and other occasions [23]; $w_{1}$ and $w_{2}$ are the weights of $\mathbf{q}_{1}$ and $\mathbf{q}_{2}$, respectively.

In terms of the architecture of information fusion, nonlinear filters are divided into centralized and decentralized types [26]. According to the above ideas, we develop four kinds of attitude estimation layouts based on EKF to estimate the attitude of CubeSat by using one gyro and two STR. They are centralized layout I, centralized layout II, centralized layout III, and decentralized layout, respectively.

3.1.1. Centralized Layout I. Figure 5 shows the structure diagram of the centralized layout I.

$\tilde{\mathbf{q}}_{b i_{-} 1}$ is the quaternion from STR $1 ; \widehat{\mathbf{b}}, \widehat{\mathbf{b}}$, and $\Delta \widehat{\mathbf{b}}$ are the estimation, prediction, and estimation error of gyro bias, respectively; $\widehat{\mathbf{q}}_{b o}$ is the predicted attitude quaternion of the orbit frame relative to the CubeSat body frame; $\widehat{\mathbf{P}}$ and $\widehat{\mathbf{P}}$ are the prediction and estimation error covariance matrix, respectively.

3.1.2. Centralized Layout II. Figure 6 shows the structure diagram of the centralized layout II.

$\tilde{\mathbf{q}}_{b i \_2}$ is the quaternion from STR 2; $\tilde{\mathbf{q}}_{b i \_w}$ is the nonlinear weighted average of $\tilde{\mathbf{q}}_{b i \_1}$ and $\tilde{\mathbf{q}}_{b i \_2}$.

3.1.3. Centralized Layout III. Figure 7 shows the structure diagram of the centralized layout III.

$\Delta \widehat{\mathbf{q}}_{b o_{-} a_{-} v}$ and $\Delta \widehat{\mathbf{q}}_{b o_{-} b_{-} v}$ are the vector parts of two estimation error quaternions from MEKF; $\Delta \widehat{\mathbf{q}}_{b_{-} w_{-} v}$ is the linear weighted average of $\Delta \widehat{\mathbf{q}}_{b o_{-} a_{-} v}$ and $\Delta \widehat{\mathbf{q}}_{b o_{-} b_{-} v}$.

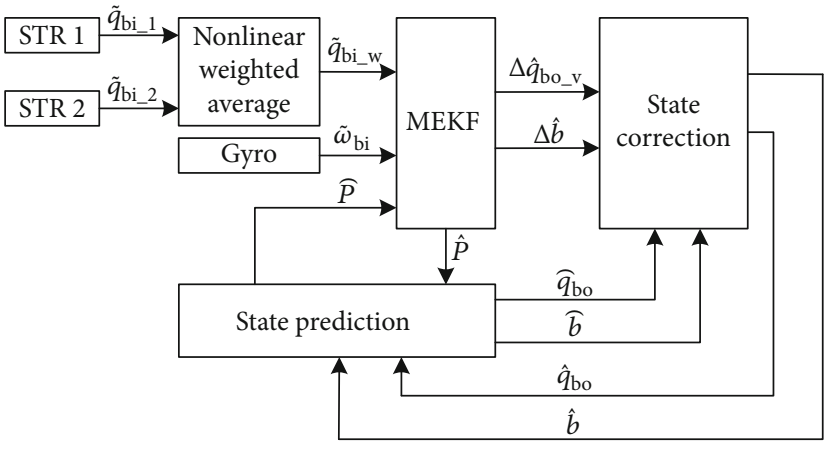

FIgure 6: The centralized layout II.

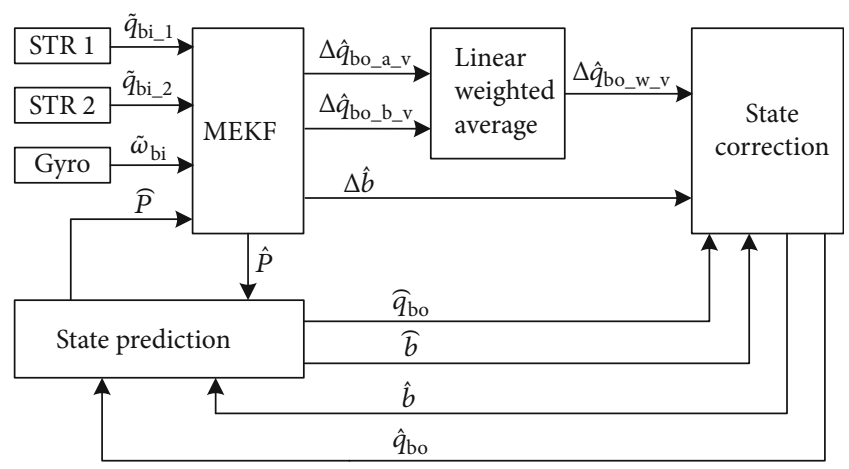

FIgURE 7: The centralized layout III.

3.1.4. Decentralized Layout. Figure 8 shows the structure diagram of the decentralized layout.

$\Delta \widehat{\mathbf{b}}_{1}$ and $\Delta \widehat{\mathbf{b}}_{2}$ are the two bias estimation errors from MEKF 1 and MEKF 2, respectively; $\Delta \widehat{\mathbf{b}}_{w}$ is the linear

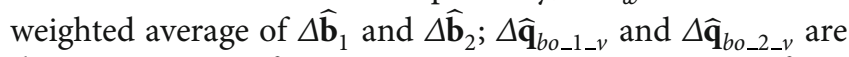
the vector parts of two estimation error quaternions from MEKF 1 and MEKF 2, respectively; $\widehat{\mathbf{P}}_{1}$ and $\widehat{\mathbf{P}}_{2}$ are the prediction estimation error covariance matrices of $\left[\begin{array}{ll}\Delta \mathbf{b} \wedge_{1}{ }^{T} & \Delta \mathbf{q} \wedge_{b o_{-}{ }_{-} v}{ }^{T}\end{array}\right]^{T} \quad$ and $\quad\left[\begin{array}{lll}\Delta \mathbf{b} \wedge_{2}{ }^{T} & \Delta \mathbf{q} \wedge_{b o_{-}{ }_{-}-}{ }^{T}\end{array}\right]^{T}$, respectively; $\widehat{\mathbf{P}}_{w}$ is the linear weighted average of $\widehat{\mathbf{P}}_{1}$ and $\widehat{\mathbf{P}}_{2}$.

\subsection{Attitude Estimation Process}

3.2.1. Prediction of State. According to the attitude kinematics model (2), gyro bias model (4b), and estimation of state at time $k-1$, the prediction of state at time $k$ can be obtained by

$$
\begin{aligned}
\widehat{\mathbf{q}}_{b o, k} & =\widehat{\mathbf{q}}_{b o, k-1}+\left(\frac{1}{2} \boldsymbol{\Omega}\left(\widehat{\boldsymbol{w}}_{b o, k-1}\right) \widehat{\mathbf{q}}_{b o, k-1}\right) \Delta t, \\
\widehat{\mathbf{b}}_{k} & =\widehat{\mathbf{b}}_{k-1},
\end{aligned}
$$

where $\Delta t$ is the filter period; $\widehat{\boldsymbol{\omega}}_{b o, k-1}$ is the estimated angular rate of the CubeSat body frame relative to the orbit frame, denoted as 


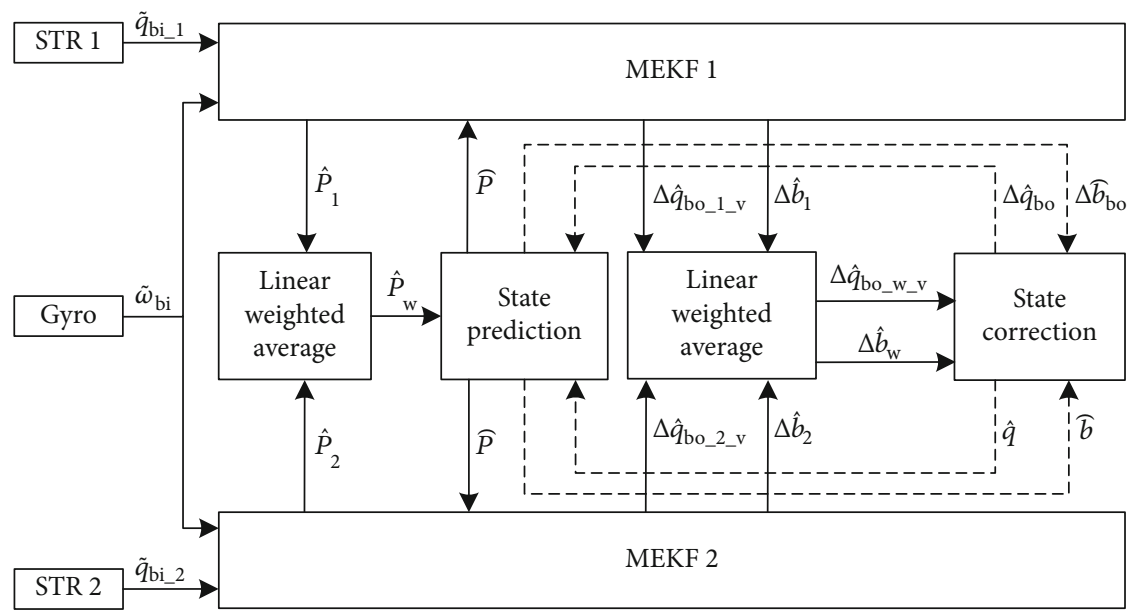

Figure 8: The decentralized layout.

$$
\widehat{\boldsymbol{\omega}}_{b o, k-1}=\tilde{\boldsymbol{\omega}}_{b i, k-1}-\widehat{\mathbf{b}}_{k-1}-\mathbf{T}\left(\widehat{\mathbf{q}}_{b o, k-1}\right) \boldsymbol{\omega}_{o i, k-1},
$$

where $\boldsymbol{\omega}_{o i, k-1}$ is the angular rate of the orbit frame relative to the geocentric inertial frame, denoted as

$$
\boldsymbol{\omega}_{o i, k-1}=\left[\begin{array}{lll}
0 & -\omega_{o, k-1} & 0
\end{array}\right]^{T},
$$

where $\omega_{o, k-1}$ is the mean orbit angular rate.

$\mathbf{T}(\mathbf{q})$ is the direction cosine matrix represented by the quaternion, denoted as

$\mathbf{T}(\mathbf{q})=\left[\begin{array}{ccc}2\left(q_{0}^{2}+q_{1}^{2}\right)-1 & 2\left(q_{1} q_{2}+q_{0} q_{3}\right) & 2\left(q_{1} q_{3}-q_{0} q_{2}\right) \\ 2\left(q_{1} q_{2}-q_{0} q_{3}\right) & 2\left(q_{0}^{2}+q_{2}^{2}\right)-1 & 2\left(q_{2} q_{3}+q_{0} q_{1}\right) \\ 2\left(q_{1} q_{3}+q_{0} q_{2}\right) & 2\left(q_{2} q_{3}-q_{0} q_{1}\right) & 2\left(q_{0}{ }^{2}+q_{3}{ }^{2}\right)-1\end{array}\right]$

3.2.2. MEKF Equation. Since the estimation of state error is identically equal to zero at the initial time of each iteration [24], MEKF is simplified as follows.

Step (1). Prediction error covariance matrix

$$
\widehat{\mathbf{P}}_{k}=\boldsymbol{\Phi}_{k / k-1} \widehat{\mathbf{P}}_{k-1} \boldsymbol{\Phi}_{k / k-1}^{T}+\boldsymbol{\Gamma}_{k / k-1} \mathbf{Q}_{k-1} \Gamma_{k / k-1}^{T}
$$

where $\boldsymbol{\Phi}_{k / k-1}$ is the state error transition matrix from time $k-1$ to time $k ; \Gamma_{k / k-1}$ is the process noise input matrix; $\mathbf{Q}_{k-1}$ is the process noise variance matrix.

(1) For the centralized layout I, centralized layout II, and decentralized layout, $\Phi_{k / k-1}$ is denoted as

$$
\boldsymbol{\Phi}_{k / k-1}=\mathbf{I}_{6 \times 6}+\mathbf{F}_{k-1} \Delta t+\mathbf{F}_{k-1}{ }^{2} \frac{\Delta t^{2}}{2 !}+\mathbf{F}_{k-1}{ }^{3} \frac{\Delta t^{3}}{3 !},
$$

where $\mathbf{F}_{k-1}$ is the Jacobian matrix with respect to state error, denoted as

$$
\mathbf{F}_{k-1}=\left[\begin{array}{cc}
\mathbf{0}_{3 \times 3} & \mathbf{0}_{3 \times 3} \\
-\frac{1}{2} \mathbf{I}_{3 \times 3} & -\left[\widehat{\boldsymbol{\omega}}_{b i, k-1} \times\right]
\end{array}\right],
$$

where $\widehat{\boldsymbol{\omega}}_{b i, k-1}$ is the estimated angular rate of the CubeSat body frame relative to the geocentric inertial frame, $\widehat{\boldsymbol{\omega}}_{b i, k-1}$ $=\tilde{\boldsymbol{\omega}}_{b i, k-1}-\widehat{\mathbf{b}}_{k-1} ;[\boldsymbol{\omega} \times]$ is denoted as

$$
[\boldsymbol{\omega} \times]=\left[\begin{array}{ccc}
0 & -\omega_{z} & \omega_{y} \\
\omega_{z} & 0 & -\omega_{x} \\
-\omega_{y} & \omega_{x} & 0
\end{array}\right]
$$

$\Gamma_{k / k-1}$ is denoted as

$$
\boldsymbol{\Gamma}_{k / k-1}=\left(\mathbf{I}_{6 \times 6} \Delta t+\mathbf{F}_{k-1} \frac{1}{2} \Delta t^{2}+\mathbf{F}_{k-1}{ }^{2} \frac{1}{6} \Delta t^{3}+\mathbf{F}_{k-1}{ }^{3} \frac{1}{24} \Delta t^{4}\right) \mathbf{G}_{k-1},
$$

where $\mathbf{G}_{k-1}$ is the Jacobian matrix with respect to process noise, denoted as

$$
\mathbf{G}_{k-1}=\left[\begin{array}{cc}
\mathbf{I}_{3 \times 3} & \mathbf{0}_{3 \times 3} \\
\mathbf{0}_{3 \times 3} & -\frac{1}{2} \mathbf{I}_{3 \times 3}
\end{array}\right] .
$$

$\mathbf{Q}_{k-1}$ is denoted as

$$
\mathbf{Q}_{k-1}=\left[\begin{array}{cc}
K^{2} T_{s} \mathbf{I}_{3 \times 3} & -\frac{1}{4} K^{2} T_{s}^{2} \mathbf{I}_{3 \times 3} \\
-\frac{1}{4} K^{2} T_{s}^{2} \mathbf{I}_{3 \times 3} & \left(\frac{1}{4} N^{2} T_{s}+\frac{1}{12} K^{2} T_{s}^{3}\right) \mathbf{I}_{3 \times 3}
\end{array}\right] .
$$


(2) For the centralized layout III, $\boldsymbol{\Phi}_{k / k-1}$ is denoted as

$$
\boldsymbol{\Phi}_{k / k-1}=\mathbf{I}_{9 \times 9}+\mathbf{F}_{k-1} \Delta t+\mathbf{F}_{k-1}{ }^{2} \frac{\Delta t^{2}}{2 !}+\mathbf{F}_{k-1}{ }^{3} \frac{\Delta t^{3}}{3 !}
$$

where $\mathbf{F}_{k-1}$ is denoted as

$$
\mathbf{F}_{k-1}=\left[\begin{array}{ccc}
\mathbf{0}_{3 \times 3} & \mathbf{0}_{3 \times 3} & \mathbf{0}_{3 \times 3} \\
-\frac{1}{2} \mathbf{I}_{3 \times 3} & -\left[\widehat{\boldsymbol{\omega}}_{b i, k-1} \times\right] & \mathbf{0}_{3 \times 3} \\
-\frac{1}{2} \mathbf{I}_{3 \times 3} & \mathbf{0}_{3 \times 3} & -\left[\widehat{\boldsymbol{\omega}}_{b i, k-1} \times\right]
\end{array}\right] .
$$

$\Gamma_{k / k-1}$ is denoted as

$$
\boldsymbol{\Gamma}_{k / k-1}=\left(\mathbf{I}_{9 \times 9} \Delta t+\mathbf{F}_{k-1} \frac{1}{2} \Delta t^{2}+\mathbf{F}_{k-1}{ }^{2} \frac{1}{6} \Delta t^{3}+\mathbf{F}_{k-1}{ }^{3} \frac{1}{24} \Delta t^{4}\right) \mathbf{G}_{k-1},
$$

where $\mathbf{G}_{k-1}$ is denoted as

$$
\mathbf{G}_{k-1}=\left[\begin{array}{ccc}
\mathbf{I}_{3 \times 3} & \mathbf{0}_{3 \times 3} & \mathbf{0}_{3 \times 3} \\
\mathbf{0}_{3 \times 3} & -\frac{1}{2} \mathbf{I}_{3 \times 3} & \mathbf{0}_{3 \times 3} \\
\mathbf{0}_{3 \times 3} & \mathbf{0}_{3 \times 3} & -\frac{1}{2} \mathbf{I}_{3 \times 3}
\end{array}\right] .
$$

$\mathbf{Q}_{k-1}$ is denoted as

$$
\mathbf{Q}_{k-1}=\left[\begin{array}{ccc}
K^{2} T_{s} \mathbf{I}_{3 \times 3} & -\frac{1}{4} K^{2} T_{s}{ }^{2} \mathbf{I}_{3 \times 3} & -\frac{1}{4} K^{2} T_{s}{ }^{2} \mathbf{I}_{3 \times 3} \\
-\frac{1}{4} K^{2} T_{s}{ }^{2} \mathbf{I}_{3 \times 3} & \left(\frac{1}{4} N^{2} T_{s}+\frac{1}{12} K^{2} T_{s}^{3}\right) \mathbf{I}_{3 \times 3} & \frac{1}{12} K^{2} T_{s}^{3} \mathbf{I}_{3 \times 3} \\
-\frac{1}{4} K^{2} T_{s}^{2} \mathbf{I}_{3 \times 3} & \frac{1}{12} K^{2} T_{s}^{3} I_{3 \times 3} & \left(\frac{1}{4} N^{2} T_{s}+\frac{1}{12} K^{2} T_{s}^{3}\right) \mathbf{I}_{3 \times 3}
\end{array}\right]
$$

Step (2). Kalman gain matrix

$$
\mathbf{K}_{k}=\widehat{\mathbf{P}}_{k} \mathbf{H}_{k}^{T}\left(\mathbf{H}_{k} \widehat{\mathbf{P}}_{k} \mathbf{H}_{k}^{T}+\mathbf{R}_{k}\right)^{-1}
$$

where $\mathbf{H}_{k}$ is the state error measurement matrix; $\boldsymbol{R}_{k}$ is the measurement noise variance matrix.

(1) For the centralized layout I,

$$
\begin{aligned}
\mathbf{H}_{k} & =\left[\begin{array}{ll}
\mathbf{0}_{3 \times 3} & \mathbf{I}_{3 \times 3}
\end{array}\right], \\
\mathbf{R}_{k} & =S_{1} \mathbf{I}_{3 \times 3},
\end{aligned}
$$

where $S_{1}$ is the variance of $\Delta \tilde{\mathbf{q}}_{b i_{-} 1} ; \Delta \tilde{\mathbf{q}}_{b i_{-} 1}$ is the converted measurement noise of STR 1 .

(2) For the centralized layout II,

$$
\begin{aligned}
& \mathbf{H}_{k}=\left[\begin{array}{ll}
\mathbf{0}_{3 \times 3} & \mathbf{I}_{3 \times 3}
\end{array}\right], \\
& \mathbf{R}_{k}=S_{w} \mathbf{I}_{3 \times 3},
\end{aligned}
$$

where $S_{w}$ is the variance of $\Delta \tilde{\mathbf{q}}_{b i \_w} ; \Delta \tilde{\mathbf{q}}_{b i \_w}$ is the converted measurement noise of STR 1 combined with STR 2.

(3) For the centralized layout III,

$$
\begin{aligned}
& \mathbf{H}_{k}=\left[\begin{array}{lll}
\mathbf{0}_{3 \times 3} & \mathbf{I}_{3 \times 3} & \mathbf{0}_{3 \times 3} \\
\mathbf{0}_{3 \times 3} & \mathbf{0}_{3 \times 3} & \mathbf{I}_{3 \times 3}
\end{array}\right], \\
& \mathbf{R}_{k}=\left[\begin{array}{cc}
S_{1} \mathbf{I}_{3 \times 3} & \mathbf{0}_{3 \times 3} \\
\mathbf{0}_{3 \times 3} & S_{2} \mathbf{I}_{3 \times 3}
\end{array}\right],
\end{aligned}
$$

where $S_{2}$ is the variance of $\Delta \tilde{\mathbf{q}}_{b i_{-} 2} ; \Delta \tilde{\mathbf{q}}_{b i_{-} 2}$ is the converted measurement noise of STR 2 .

(4) For the decentralized layout,

$$
\begin{aligned}
& \mathbf{H}_{1, k}=\left[\begin{array}{ll}
\mathbf{0}_{3 \times 3} & \mathbf{I}_{3 \times 3}
\end{array}\right], \\
& \mathbf{H}_{2, k}=\left[\begin{array}{ll}
\mathbf{0}_{3 \times 3} & \mathbf{I}_{3 \times 3}
\end{array}\right], \\
& \mathbf{R}_{1, k}=S_{1} \mathbf{I}_{3 \times 3}, \\
& \mathbf{R}_{2, k}=S_{2} \mathbf{I}_{3 \times 3} .
\end{aligned}
$$

Step (3). Estimation of state error

$$
\Delta \widehat{\mathbf{X}}_{k}=\mathbf{K}_{k} \delta \mathbf{Z}_{k},
$$

where $\delta \mathbf{Z}_{k}$ is the integrated measurement residual.

(1) For the centralized layout I, 


$$
\begin{aligned}
\Delta \widehat{\mathbf{X}}_{k} & =\left[\begin{array}{ll}
\Delta \mathbf{b} \wedge_{k}{ }^{T} & \Delta \mathbf{q} \wedge_{b o_{-} v, k}{ }^{T}
\end{array}\right]^{T}, \\
\delta \mathbf{Z}_{k} & =\delta \tilde{\mathbf{q}}_{b i_{-} 1_{-}, k},
\end{aligned}
$$

where $\delta \tilde{\mathbf{q}}_{b i \_1 \_v, k}$ is the measurement residual of STR 1 .

(2) For the centralized layout II,

$$
\begin{aligned}
\Delta \widehat{\mathbf{X}}_{k} & =\left[\begin{array}{ll}
\Delta \mathbf{b} \wedge_{k}^{T} & \Delta \mathbf{q} \wedge_{b o_{-} v, k}{ }^{T}
\end{array}\right]^{T}, \\
\delta \mathbf{Z}_{k} & =\delta \tilde{\mathbf{q}}_{b i \_w \_v, k},
\end{aligned}
$$

where $\delta \tilde{\mathbf{q}}_{b i \_w \_v, k}$ is the measurement residual of STR 1 combined with STR 2.

(3) For the centralized layout III,

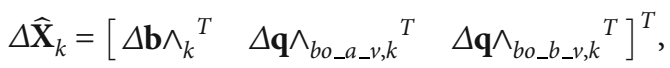

$$
\begin{aligned}
& \delta \mathbf{Z}_{k}=\left[\begin{array}{ll}
\delta \tilde{\mathbf{q}}_{b i \_1 \_v, k} & \delta \tilde{\mathbf{q}}_{b i \_2 \_v, k}
\end{array}\right]^{T},
\end{aligned}
$$

where $\delta \tilde{\mathbf{q}}_{b i \_2 \_v, k}$ is the measurement residual of STR 2.

(4) For the decentralized layout,

$$
\begin{aligned}
\Delta \widehat{\mathbf{X}}_{1, k} & =\left[\begin{array}{ll}
\Delta \mathbf{b} \wedge_{1, k}^{T} & \Delta \mathbf{q} \wedge_{b o_{-} 1 \_v, k}^{T}
\end{array}\right]^{T}, \\
\Delta \widehat{\mathbf{X}}_{2, k} & =\left[\begin{array}{ll}
\Delta \mathbf{b} \wedge_{2, k}^{T} & \Delta \mathbf{q} \wedge_{b o \_2 \_v, k}^{T}
\end{array}\right]^{T}, \\
\delta \mathbf{Z}_{1, k} & =\delta \tilde{\mathbf{q}}_{b i \_1 \_v, k}, \\
\delta \mathbf{Z}_{2, k} & =\delta \tilde{\mathbf{q}}_{b i \_2 \_v, k} .
\end{aligned}
$$

Step (4). Estimation error covariance matrix.

(1) For the centralized layout I and centralized layout II,

$$
\widehat{\mathbf{P}}_{k}=\left(\mathbf{I}_{6 \times 6}-\mathbf{K}_{k} \mathbf{H}_{k}\right) \widehat{\mathbf{P}}_{k}\left(\mathbf{I}_{6 \times 6}-\mathbf{K}_{k} \mathbf{H}_{k}\right)^{T}+\mathbf{K}_{k} \mathbf{R}_{k} \mathbf{K}_{k}^{T}
$$

(2) For the centralized layout III,

$$
\begin{aligned}
\widehat{\mathbf{P}}_{k} & =\left(\mathbf{I}_{9 \times 9}-\mathbf{K}_{k} \mathbf{H}_{k}\right) \widehat{\mathbf{P}}_{k}\left(\mathbf{I}_{9 \times 9}-\mathbf{K}_{k} \mathbf{H}_{k}\right)^{T}+\mathbf{K}_{k} \mathbf{R}_{k} \mathbf{K}_{k}{ }^{T}, \\
\widehat{\mathbf{P}}_{w, k} & =\left(\mathbf{P} \wedge_{a, k}{ }^{-1}+\mathbf{P} \wedge_{b, k}{ }^{-1}\right)^{-1},
\end{aligned}
$$

where $\widehat{\mathbf{P}}_{a, k}$ and $\widehat{\mathbf{P}}_{b, k}$ are the estimation error covariance matrices of $\Delta \widehat{\mathbf{q}}_{b o_{\_} a_{-} v, k}$ and $\Delta \widehat{\mathbf{q}}_{b o_{-} b_{-} v, k}$, respectively, in $\widehat{\mathbf{P}}_{k}$.

(3) For the decentralized layout,
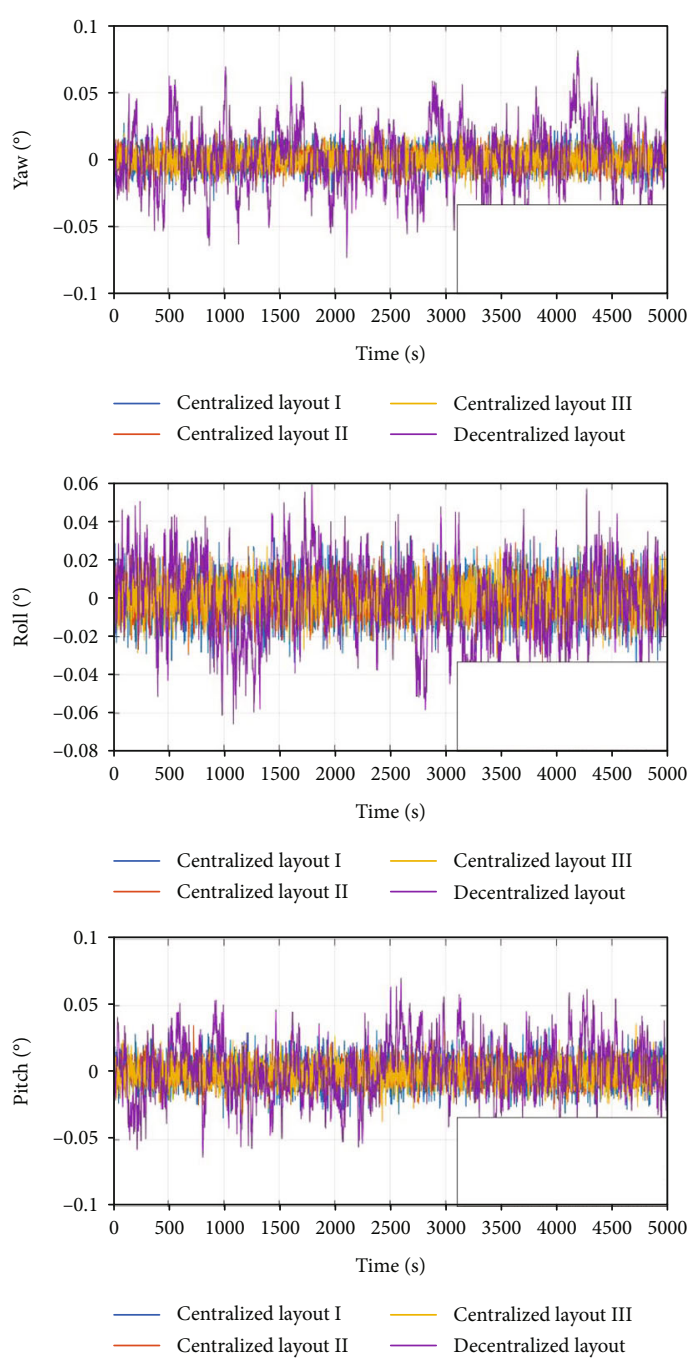

FIgURE 9: Estimation error of Euler angles (stable phase).

$\widehat{\mathbf{P}}_{1, k}=\left(\mathbf{I}_{6 \times 6}-\mathbf{K}_{1, k} \mathbf{H}_{1, k}\right) \widehat{\mathbf{P}}_{k}\left(\mathbf{I}_{6 \times 6}-\mathbf{K}_{1, k} \mathbf{H}_{1, k}\right)^{T}+\mathbf{K}_{1, k} \mathbf{R}_{1, k} \mathbf{K}_{1, k}{ }^{T}$,

$$
\begin{aligned}
& \widehat{\mathbf{P}}_{2, k}=\left(\mathbf{I}_{6 \times 6}-\mathbf{K}_{2, k} \mathbf{H}_{2, k}\right) \widehat{\mathbf{P}}_{k}\left(\mathbf{I}_{6 \times 6}-\mathbf{K}_{2, k} \mathbf{H}_{2, k}\right)^{T}+\mathbf{K}_{2, k} \mathbf{R}_{2, k} \mathbf{K}_{2, k}{ }^{T}, \\
& \widehat{\mathbf{P}}_{w, k}=\left(\mathbf{P} \wedge_{1, k}{ }^{-1}+\mathbf{P} \wedge_{2, k}{ }^{-1}\right)^{-1} .
\end{aligned}
$$

\subsubsection{Correction of State}

(1) For the centralized layout I and centralized layout II,

$$
\begin{aligned}
\widehat{\mathbf{q}}_{b o, k} & =\widehat{\mathbf{q}}_{b o, k} \otimes\left[\sqrt{1-\left\|\Delta \mathbf{q} \wedge_{b o_{-} v, k}\right\|^{2}} \quad \Delta \mathbf{q} \wedge_{b o_{-} v, k}\right]^{T}, \\
\widehat{\mathbf{b}}_{k} & =\widehat{\mathbf{b}}_{k}+\Delta \widehat{\mathbf{b}}_{k} .
\end{aligned}
$$

(2) For the centralized layout III, 
TABLE 2: RMSE and MAE of estimation error of Euler angles.

\begin{tabular}{|c|c|c|c|c|c|}
\hline \multicolumn{2}{|c|}{$\begin{array}{l}\text { Attitude estimation } \\
\text { layouts } \\
\text { Evaluation index }\end{array}$} & Centralized layout I & Centralized layout II & Centralized layout III & Decentralized layout \\
\hline \multirow{2}{*}{ Yaw } & $\operatorname{RMSE}\left({ }^{\circ}\right)$ & 0.00781 & 0.00633 & 0.00695 & 0.02085 \\
\hline & $\operatorname{MAE}\left({ }^{\circ}\right)$ & 0.00624 & 0.00506 & 0.00553 & 0.01677 \\
\hline \multirow{2}{*}{ Roll } & $\operatorname{RMSE}\left(\left(^{\circ}\right)\right.$ & 0.01024 & 0.00803 & 0.00867 & 0.02266 \\
\hline & $\operatorname{MAE}\left({ }^{\circ}\right)$ & 0.00816 & 0.00641 & 0.00689 & 0.01809 \\
\hline \multirow{2}{*}{ Pitch } & $\operatorname{RMSE}\left({ }^{\circ}\right)$ & 0.01034 & 0.00824 & 0.00852 & 0.02120 \\
\hline & $\operatorname{MAE}\left(^{\circ}\right)$ & 0.00827 & 0.00657 & 0.00680 & 0.01684 \\
\hline
\end{tabular}

$$
\begin{aligned}
& \Delta \widehat{\mathbf{q}}_{b o_{\_} \omega_{-} v, k}=\widehat{\mathbf{P}}_{w, k}\left(\mathbf{P} \wedge_{a, k}{ }^{-1} \Delta \widehat{\mathbf{q}}_{b o_{\_} a_{-} v, k}+\mathbf{P} \wedge_{b, k}{ }^{-1} \Delta \widehat{\mathbf{q}}_{b o_{\_} b_{-} v, k}\right), \\
& \widehat{\mathbf{q}}_{b o, k}=\widehat{\mathbf{q}}_{b o, k} \otimes\left[\sqrt{1-\left\|\Delta \mathbf{q} \wedge_{b o \_} \omega_{-} v, k\right\|^{2}} \quad \Delta \mathbf{q} \wedge_{b o_{\_} \omega_{-} v, k}\right]^{T}, \\
& \widehat{\mathbf{b}}_{k}=\widehat{\mathbf{b}}_{k}+\Delta \widehat{\mathbf{b}}_{k} \text {. }
\end{aligned}
$$

(3) For the decentralized layout,

$$
\begin{aligned}
& \Delta \widehat{\mathbf{X}}_{w, k}=\widehat{\mathbf{P}}_{w, k}\left(\mathbf{P} \wedge_{1, k}^{-1} \Delta \widehat{\mathbf{X}}_{1, k}+\mathbf{P} \wedge_{2, k}{ }^{-1} \Delta \widehat{\mathbf{X}}_{2, k}\right) \\
& \Delta \widehat{\mathbf{q}}_{b o \_\omega \_v, k}=\Delta \widehat{\mathbf{X}}_{w, k}(4: 6), \\
& \widehat{\mathbf{q}}_{b o, k}=\widehat{\mathbf{q}}_{b o, k} \otimes\left[\sqrt{1-\left\|\Delta \mathbf{q} \wedge_{b o_{-} \omega_{-} v, k}\right\|^{2}} \quad \Delta \mathbf{q} \wedge_{b o_{-} \omega_{-} v, k}{ }^{T}\right]^{T}, \\
& \Delta \widehat{\mathbf{b}}_{w, k}=\Delta \widehat{\mathbf{X}}_{w, k}(1: 3), \\
& \widehat{\mathbf{b}}_{k}=\widehat{\mathbf{b}}_{k}+\Delta \widehat{\mathbf{b}}_{w, k} .
\end{aligned}
$$

\section{Simulation and Analysis}

\subsection{Simulation Parameters}

4.1.1. Navigation Parameters. The TLE for numerical simulation is as follows.

(1) 43156U 18008B 18116.45379836.00001071 00000-0 69869-4 09999

(2) 4315697.5309191 .77670014159325 .002391 .3114 15.0992586414673

The position and velocity of CubeSat at any moment are from the Simplified General Perturbations 4 (SGP4) propagator in MATLAB based on the above TLE [27].

4.1.2. Gyro Parameters. Initial true bias $\overline{\mathbf{b}}_{0}=$ $\left[\begin{array}{lll}10 & 10 & 10\end{array}\right]^{T_{\circ}} / \mathrm{h}$; initial estimated bias $\widehat{\mathbf{b}}_{0}=\left[\begin{array}{lll}0 & 0 & 0\end{array}\right]^{T_{\circ}} /$ $\mathrm{h} ; N=0.48^{\circ} / \boldsymbol{h}^{1 / 2} ; K=120.34^{\circ} / \boldsymbol{h}^{3 / 2} ; T_{s}=1$ s.
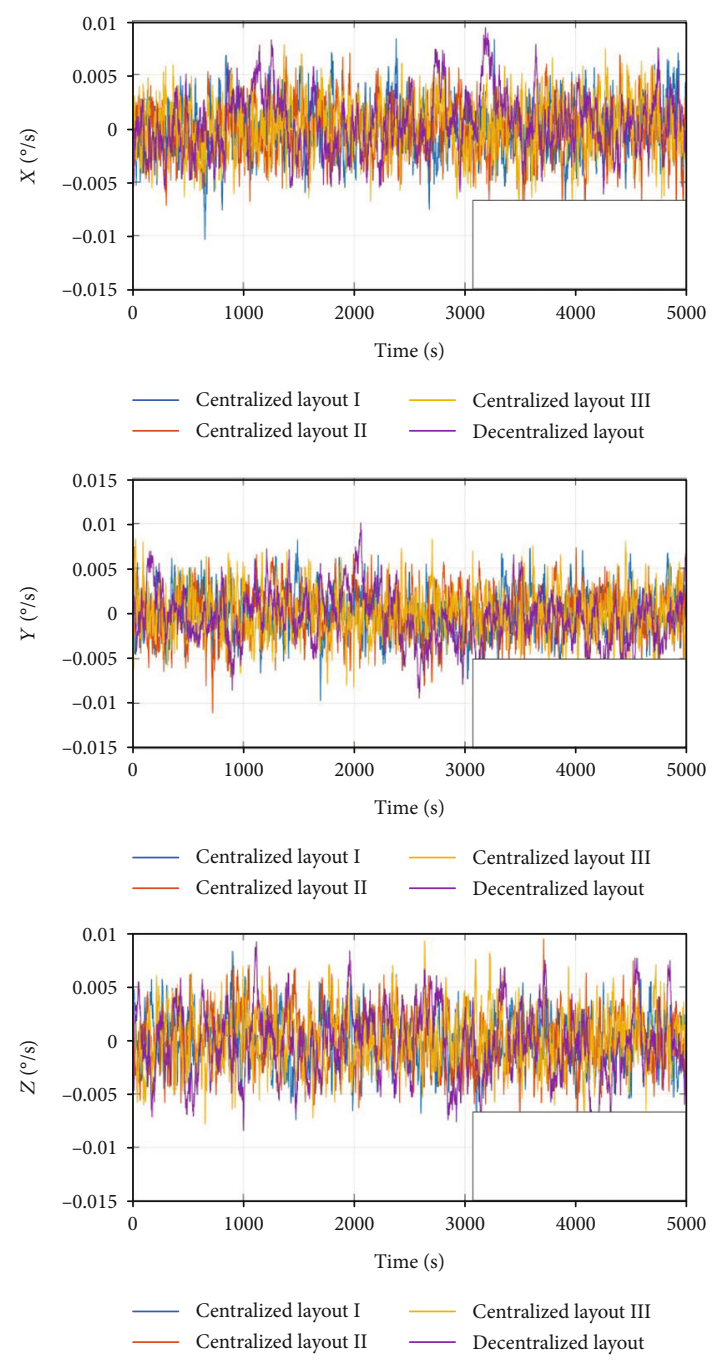

Figure 10: Estimation error of gyro biases (stable phase).

4.1.3. STR Parameters. $\Delta r_{1}=70^{\prime \prime}, \Delta p_{1}=10^{\prime \prime}, \psi_{b s_{-} 1}=-90^{\circ}$, $\varphi_{b s_{-} 1}=45^{\circ}, \theta_{b s_{-} 1}=-90^{\circ}, w_{1}=0.5, S_{1}=1.44 \times 10^{-8} ; \Delta r_{2}=70^{\prime \prime}$, $\Delta p_{2}=10^{\prime \prime}, \psi_{b s \_2}=90^{\circ}, \varphi_{b s \_2}=45^{\circ}, \theta_{b s \_2}=90^{\circ}, w_{2}=0.5, S_{2}=$ $1.44 \times 10^{-8} ; S_{w}=7.4 \times 10^{-9}$.

4.1.4. Initial Attitude Knowledge. True Euler angle $\overline{\mathbf{a}}_{b o, 0}=$ $\left[\begin{array}{lll}5 & -5 & 5\end{array}\right]^{\circ}$; estimated Euler angle $\widehat{\mathbf{a}}_{b o, 0}=\left[\begin{array}{lll}0 & 0 & 0\end{array}\right]^{\circ}$ 
TABLE 3: RMSE and MAE of estimation error of gyro biases.

\begin{tabular}{|c|c|c|c|c|c|}
\hline \multicolumn{2}{|c|}{$\begin{array}{l}\text { Attitude estimation } \\
\text { layouts } \\
\text { Evaluation index }\end{array}$} & Centralized layout I & Centralized layout II & Centralized layout III & Decentralized layout \\
\hline$X$ & $\operatorname{RMSE}(\% / \mathrm{s})$ & 0.00251 & 0.00237 & 0.00240 & 0.00321 \\
\hline$\lambda$ & $\operatorname{MAE}(\%)$ & 0.00203 & 0.00189 & 0.00191 & 0.00249 \\
\hline \multirow{2}{*}{$Y$} & $\operatorname{RMSE}(\% / s)$ & 0.00247 & 0.00235 & 0.00242 & 0.00283 \\
\hline & $\operatorname{MAE}(\% / \mathrm{s})$ & 0.00197 & 0.00190 & 0.00193 & 0.00226 \\
\hline \multirow{2}{*}{$Z$} & $\operatorname{RMSE}(\% / s)$ & 0.00241 & 0.00231 & 0.00237 & 0.00284 \\
\hline & $\operatorname{MAE}(\% / \mathrm{s})$ & 0.00190 & 0.00186 & 0.00187 & 0.00234 \\
\hline
\end{tabular}

$; \overline{\mathbf{q}}_{b o, 0}=\left[\begin{array}{llll}0.9972 & -0.0454 & 0.0416 & 0.0416\end{array}\right]^{T} ; \quad \widehat{\mathbf{q}}_{b o, 0}=$ $\left[\begin{array}{llll}1 & 0 & 0 & 0\end{array}\right]^{T}$; true Euler angular rate $\overline{\mathbf{r}}_{b o, 0}=\left[\begin{array}{lll}-1 & 1 & 1\end{array}\right]^{\circ}$ /s; estimated Euler angular rate $\widehat{\mathbf{r}}_{b o, 0}=\left[\begin{array}{lll}0 & 0 & 0\end{array}\right]^{\circ} / \mathrm{s}$.

4.1.5. Initial Estimation Error Covariance Matrix. For the centralized layout I, centralized layout II, and decentralized layout, $\widehat{\mathbf{P}}_{0}=\mathbf{I}_{6 \times 6}$; for the centralized layout III, $\widehat{\mathbf{P}}_{0}=$ $\mathbf{I}_{9 \times 9}$.

4.1.6. True State Update. $\overline{\mathbf{q}}_{b o, k+1}$ used for comparison with estimated attitude quaternion is updated by

$$
\overline{\mathbf{q}}_{b o, k+1}=\overline{\mathbf{q}}_{b o, k}+\left(\frac{1}{2} \boldsymbol{\Omega}\left(\overline{\boldsymbol{\omega}}_{b o, k}\right) \overline{\mathbf{q}}_{b o, k}\right) \Delta t,
$$

where $\overline{\boldsymbol{\omega}}_{b o, k}$ is the true angular rate of the CubeSat body frame relative to the orbit frame, denoted as

$$
\overline{\boldsymbol{\omega}}_{b o, k}=\overline{\boldsymbol{\omega}}_{b i, k}-\mathbf{T}\left(\overline{\mathbf{q}}_{b o, k}\right) \boldsymbol{\omega}_{o i, k},
$$

where $\overline{\boldsymbol{\omega}}_{b i, k}$ is denoted as

$$
\begin{aligned}
\overline{\boldsymbol{\omega}}_{b i, k}= & \overline{\boldsymbol{\omega}}_{b i, k-1}+\mathbf{I}^{-1}\left(\left[\overline{\boldsymbol{\omega}}_{b i, k-1} \times\right]\left(\mathbf{I} \overline{\boldsymbol{\omega}}_{b i, k-1}+\mathbf{h}_{r, k-1}\right)\right. \\
& \left.+\mathbf{T}_{c, k-1}+\mathbf{T}_{m, k-1}+\mathbf{T}_{a, k-1}+\mathbf{T}_{g, k-1}\right),
\end{aligned}
$$

where I is the moment of inertia of CubeSat; its value is

$$
\mathbf{I}=\left[\begin{array}{ccc}
0.003735 & -0.000001755 & -0.00004026 \\
-0.000001755 & 0.01282 & 0.00001191 \\
-0.00004026 & 0.00001191 & 0.01273
\end{array}\right]
$$

$\mathbf{h}_{r, k-1}$ is the angular momentum of reaction wheels; $\mathbf{T}_{c, k-1}$ is the control torque derived from zero-momentum attitude control [28]; $\mathbf{T}_{m, k-1}, \mathbf{T}_{a, k-1}$, and $\mathbf{T}_{g, k-1}$ are the disturbance torques derived from residual magnetism, atmospheric drag, and gravity gradient, respectively.

$\overrightarrow{\mathbf{b}}_{k+1}$ used for comparison with estimated gyro bias is updated by

$$
\overline{\mathbf{b}}_{k+1}=\overline{\mathbf{b}}_{k}+K T_{s}^{1 / 2} \boldsymbol{\eta} .
$$
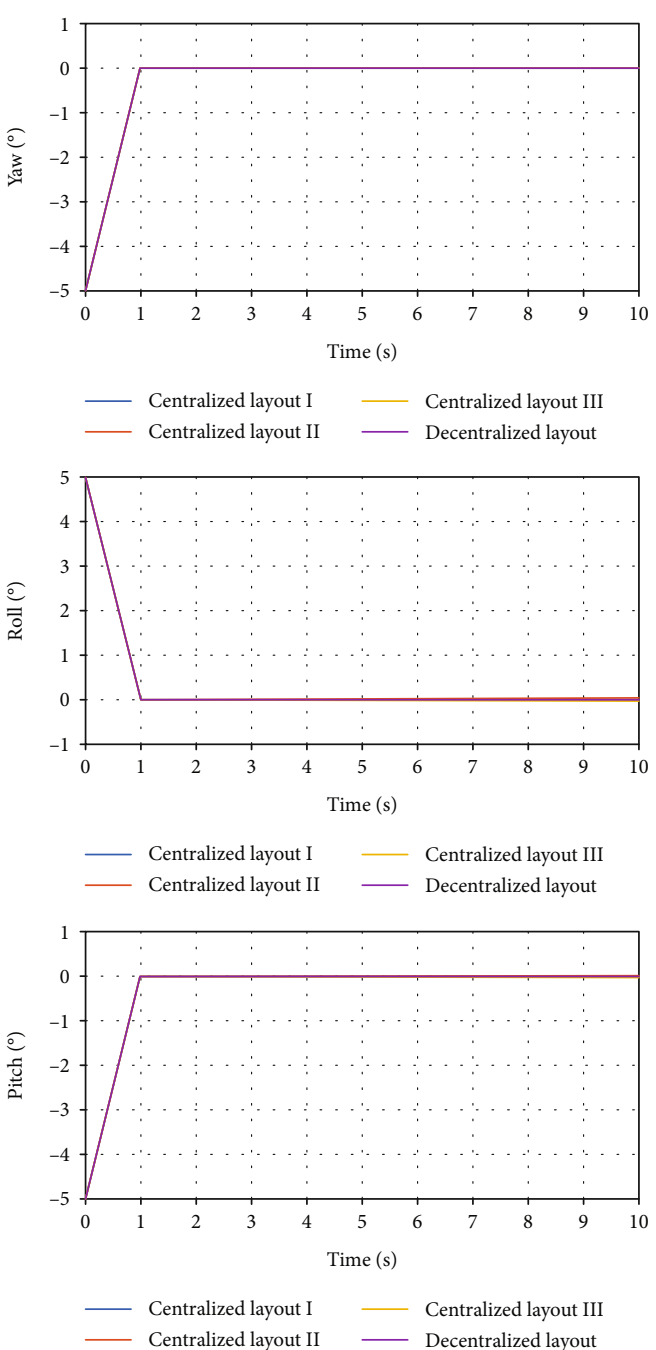

FIgURE 11: Estimation error of Euler angles (initial phase).

4.1.7. Simulation Time. Start time $t_{0}=26$ Apr $201810: 53$ : 28.178 UTCG; duration $t_{d}=5000 \mathrm{~s} ; \Delta t=1 \mathrm{~s}$.

4.1.8. Simulation Condition. Intel Core i5-4590 $3.3 \mathrm{GHz}$, dual-core, RAM 8GB; MATLAB R2015a. 
TABLe 4: Convergence time.

\begin{tabular}{lcccc}
\hline Attitude estimation layouts & Centralized layout I & Centralized layout II & Centralized layout III & Decentralized layout \\
\hline Convergence time $(\mathrm{s})$ & 1 & 1 & 1 & 1 \\
\hline
\end{tabular}

TABle 5: Execution time.

\begin{tabular}{lcccc}
\hline Attitude estimation layouts & Centralized layout I & Centralized layout II & Centralized layout III & Decentralized layout \\
\hline Execution time $(\mathrm{s})$ & 22.66 & 23.11 & 29.38 & 23.91 \\
\hline
\end{tabular}

4.2. Simulation Results 1 . The simulation and performance comparison of the above four kinds of attitude estimation layouts are carried out in terms of accuracy, convergence, and execution time.

4.2.1. Estimation Accuracy of State. Figure 9 shows the estimation error of Euler angles in a stable phase, and Table 2 lists the RMSE and MAE of the above data. We can see that the RMSE and MAE of centralized layout II are all smallest among the four layouts, which means that the centralized layout II gives the most accurate attitude knowledge with an accuracy of $0.01989^{\circ}(3 \sigma), 0.02409^{\circ}(3 \sigma)$, and $0.02472^{\circ}$ $(3 \sigma)$ in the direction of yaw, roll, and pitch, respectively.

Figure 10 shows the estimation error of gyro biases in a stable phase, and Table 3 lists the RMSE and MAE of the above data. We can see that the RMSE and MAE of centralized layout II are all smallest among the four layouts, which means that the centralized layout II gives the most accurate bias knowledge with an accuracy of $0.00711 \%(3 \sigma)$, $0.00705^{\circ} / \mathrm{s}(3 \sigma)$, and $0.00693^{\circ} / \mathrm{s}(3 \sigma)$ in the direction of $X, Y$, and $Z$, respectively.

4.2.2. Convergence Speed. Figure 11 shows the estimation error of Euler angles in the initial phase, and Table 4 lists the convergence time. We can see that the convergence time of four kinds of attitude estimation layouts is almost the same and the convergence speed is fast enough.

4.2.3. Execution Time. Table 5 lists the execution time of 5000 iterations under the same simulation condition. We can see that the execution time of centralized layout III is the longest among the four layouts.

4.3. Simulation Results 2. Furthermore, we also conduct the simulation under the same conditions for attitude measurements based on FEKF by using three STR presented in Ref. [19] and FUKF by using two STR presented in Ref. [20], respectively; their results are compared with the decentralized layout.

4.3.1. Estimation Accuracy of State. Figure 12 shows the estimation error of Euler angles in a stable phase, and Table 6 lists the RMSE and MAE of the above data. We can see that the attitude estimation accuracy of the decentralized layout is a little lower than FUKF with two STR but higher than FEKF with three STR.

Figure 13 shows the estimation error of gyro biases in a stable phase, and Table 7 lists the RMSE and MAE of the above data. We can see that the bias estimation accuracy of
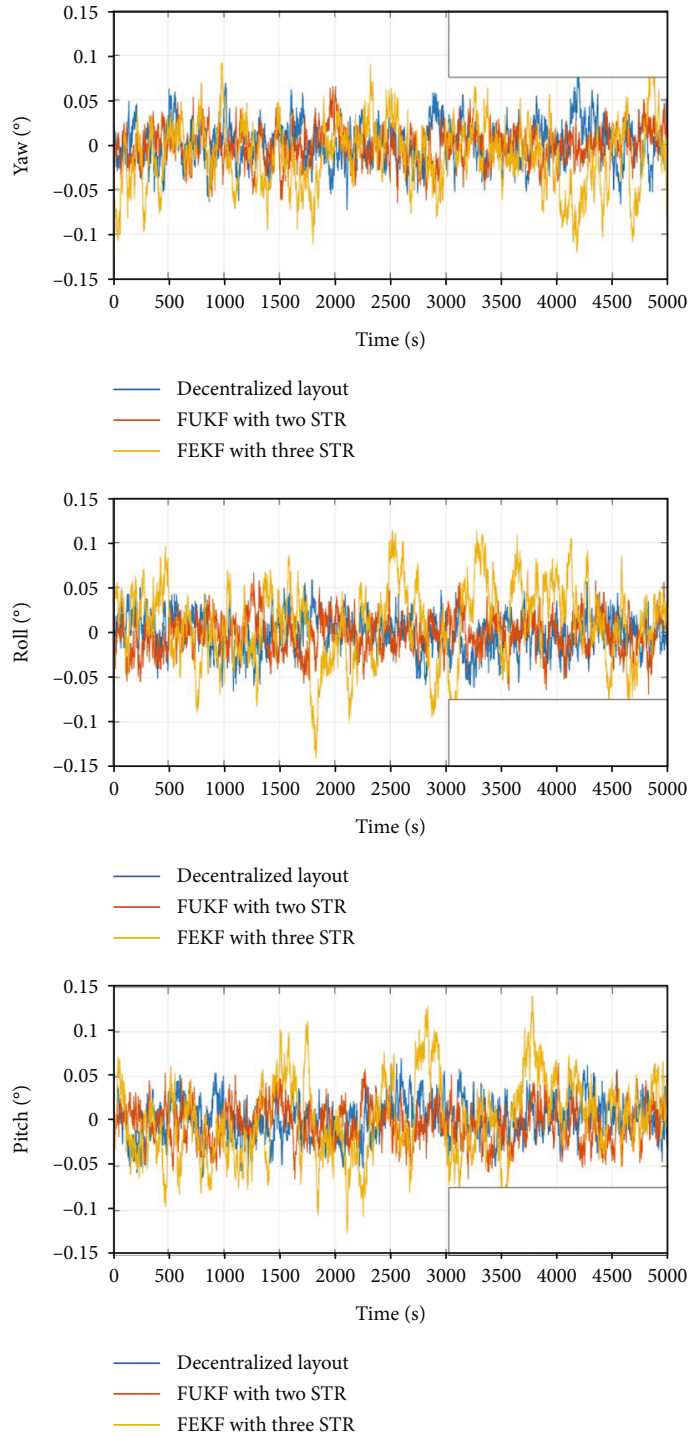

Figure 12: Estimation error of Euler angles (stable phase).

the decentralized layout is a little lower than FUKF with two STR but higher than FEKF with three STR.

4.3.2. Execution Time. Table 8 lists the execution time of 5000 iterations under the same simulation condition. We can see that the execution time of FUKF with two STR is the longest among the three layouts, and it is more than twice that of the decentralized layout. 
TABLE 6: RMSE and MAE of estimation error of Euler angles.

\begin{tabular}{lcccc}
\hline $\begin{array}{l}\text { Attitude } \\
\text { estimation layouts } \\
\text { Evaluation index }\end{array}$ & $\begin{array}{c}\text { Decentralized } \\
\text { layout }\end{array}$ & $\begin{array}{c}\text { FUKF } \\
\text { with } \\
\text { two STR }\end{array}$ & $\begin{array}{c}\text { FEKF } \\
\text { with } \\
\text { three STR }\end{array}$ \\
\hline Yaw & $\begin{array}{c}\text { RMSE } \\
\left(^{\circ}\right) \\
\text { MAE }\left(^{\circ}\right)\end{array}$ & 0.02085 & 0.01965 & 0.03805 \\
\hline & $\begin{array}{c}\text { RMSE } \\
\left(^{\circ}\right)\end{array}$ & 0.01677 & 0.01596 & 0.02970 \\
\hline Roll & $\operatorname{MAE~}\left(^{\circ}\right)$ & 0.01809 & 0.01692 & 0.03585 \\
\hline & $\begin{array}{c}\text { RMSE } \\
\left(^{\circ}\right)\end{array}$ & 0.02120 & 0.02023 & 0.04373 \\
Pitch & $\operatorname{MAE~}\left(^{\circ}\right)$ & 0.01684 & 0.01644 & 0.03505 \\
\hline
\end{tabular}
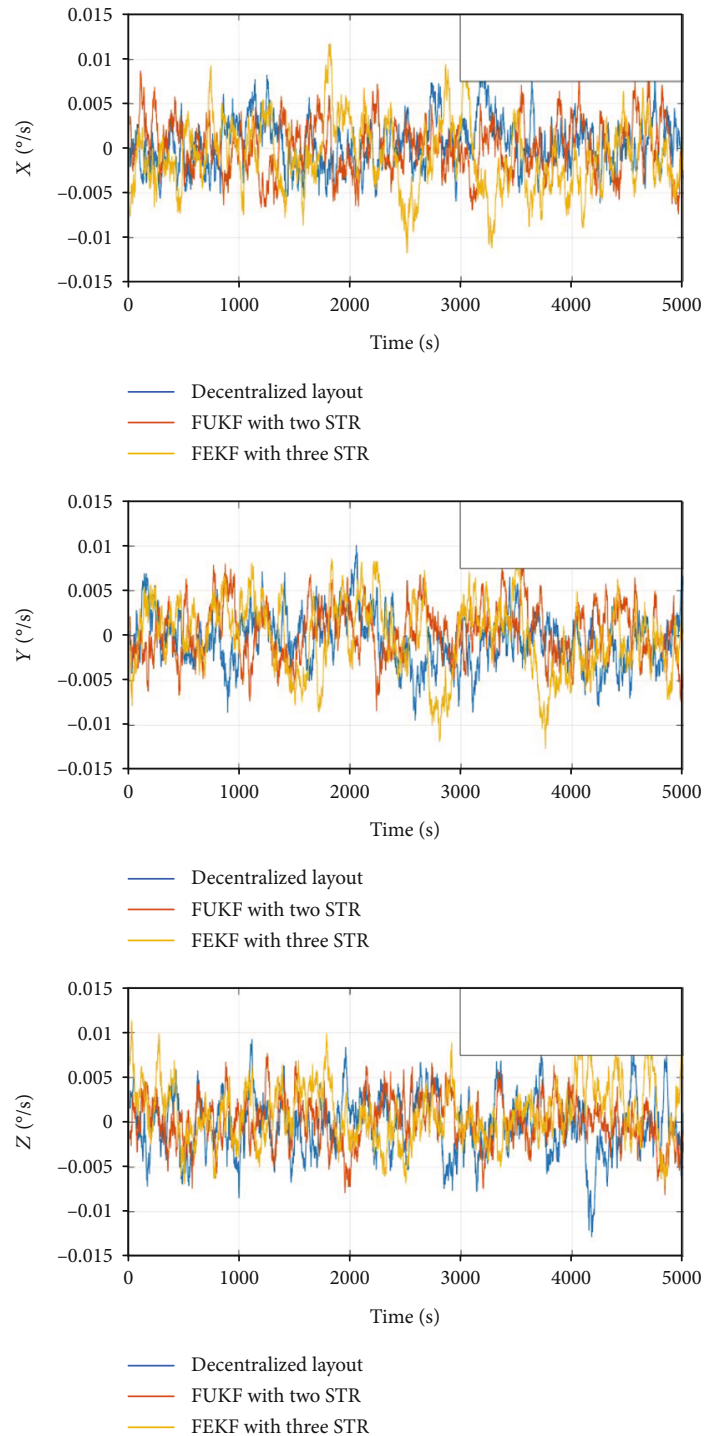

Figure 13: Estimation error of gyro biases (stable phase).
TABLE 7: RMSE and MAE of estimation error of gyro biases.

\begin{tabular}{|c|c|c|c|c|}
\hline \multicolumn{2}{|c|}{$\begin{array}{l}\text { Attitude } \\
\text { estimation } \\
\text { layouts } \\
\text { Evaluation index }\end{array}$} & $\begin{array}{l}\text { Decentralized } \\
\text { layout }\end{array}$ & $\begin{array}{c}\text { FUKF } \\
\text { with } \\
\text { two STR }\end{array}$ & $\begin{array}{c}\text { FEKF } \\
\text { with } \\
\text { three STR }\end{array}$ \\
\hline \multirow[t]{2}{*}{$X$} & $\begin{array}{c}\text { RMSE } \\
(\% / s)\end{array}$ & 0.00321 & 0.00295 & 0.00389 \\
\hline & $\operatorname{MAE}(\% / \mathrm{s})$ & 0.00249 & 0.00217 & 0.00315 \\
\hline \multirow[t]{2}{*}{ Y } & $\begin{array}{c}\text { RMSE } \\
(\% / s)\end{array}$ & 0.00283 & 0.00277 & 0.00396 \\
\hline & $\operatorname{MAE}(\% / \mathrm{s})$ & 0.00226 & 0.00216 & 0.00323 \\
\hline \multirow[t]{2}{*}{$Z$} & $\begin{array}{c}\text { RMSE } \\
(\% / s)\end{array}$ & 0.00284 & 0.00284 & 0.00329 \\
\hline & $\operatorname{MAE}(\% / s)$ & 0.00234 & 0.00232 & 0.00259 \\
\hline
\end{tabular}

TABle 8: Execution time.

\begin{tabular}{lccc}
\hline $\begin{array}{l}\text { Attitude } \\
\text { estimation layouts }\end{array}$ & $\begin{array}{c}\text { Decentralized } \\
\text { layout }\end{array}$ & $\begin{array}{c}\text { FUKF with } \\
\text { two STR }\end{array}$ & $\begin{array}{c}\text { FEKF with } \\
\text { three STR }\end{array}$ \\
\hline Execution time (s) & 23.91 & 50.99 & 30.28 \\
\hline
\end{tabular}

\section{Conclusion}

This paper proposes four kinds of attitude estimation layouts with one MEMS gyro and two simultaneously operating STR as part of the development task of the CubeSat NJUST-2. Simulation results show that the centralized layout II gives the highest estimation accuracy of Euler angles and gyro biases compared with the other three layouts and has high execution speed and fast convergence at the same time. Moreover, the comparisons between decentralized layout, FUKF with two STR, and FEKF with three STR indicate that the FUKF with two STR performs only a little better than the decentralized layout in terms of estimation accuracy but the execution time of the former is much longer than that of the latter; the attitude estimation layout with three STR cannot provide more accurate attitude and bias knowledge than that with two STR when using the same filter, and utilizing three STR will increase cost and take up more space in practical engineering application. Therefore, the centralized layout II is the best choice for the attitude measurement unit of the CubeSat with requirements of high accuracy, low cost, and limited computing capacity.

\section{Data Availability}

All data included in this study are available upon request by contacting the corresponding author.

\section{Conflicts of Interest}

The authors declare that they have no conflicts of interest. 


\section{Acknowledgments}

This work was supported in part by the National Natural Science Foundation of China (No. 61803204), the Natural Science Foundation of Jiangsu Province (No. BK20180465), and the China Scholarship Council (CSC).

\section{References}

[1] T. Villela, C. A. Costa, A. M. Brandão, F. T. Bueno, and R. Leonardi, "Towards the Thousandth CubeSat: A Statistical Overview," International Journal of Aerospace Engineering, vol. 2019, 13 pages, 2019.

[2] V. Carrara, R. B. Januzi, D. H. Makita, L. F. . P. Santos, and L. S. Sato, "The ITASAT CubeSat development and design," Journal of Aerospace Technology and Management, vol. 9, no. 2, pp. 147-156, 2017.

[3] E. L. Shkolnik, "On the verge of an astronomy CubeSat revolution," Nature Astronomy, vol. 2, no. 5, pp. 374-378, 2018.

[4] D.-H. Cho, W.-S. Choi, M.-K. Kim, J.-H. Kim, E. Sim, and H.D. Kim, "High-Resolution Image and Video CubeSat (HiREV): Development of Space Technology Test Platform Using a Low-Cost CubeSat Platform," International Journal of Aerospace Engineering, vol. 2019, 17 pages, 2019.

[5] S. Gaisser, U. Mohr, J. Keim, D. Triloff, and S. Klinkner, Improvements in attitude determination and control of the small satellite Flying Laptop, 33rd Annual AIAA/USUConference on Small Satellites, Logan, UT, USA, 2019.

[6] H. Kawasaki, K. Konoue, H. Hoshino, Y. Kaneko, and M. Sasaki, "Interim report of super low altitude satellite operation," in 2018 IEEE International Geoscience and Remote Sensing Symposium, pp. 4066-4069, Valencia, Spain, July 2018.

[7] M. F. Barschke, P. Werner, K. Gordon et al., "Initial results from the TechnoSat in-orbit demonstration mission," in 32nd Annual AIAA/USUConference on Small Satellites, pp. 126-136, AIAA, Logan, UT, USA, 2018.

[8] W. Halle, T. Terzibaschian, A. Kotz, and M. Hetscher, "Technological experiments on the DLR-BIROS satellite for the next generation of earth observation missions," in 68th International Astronautical Congress, pp. 485-492, IAC, Adelaide, Australia, 2017.

[9] Z. H. Liang, W. H. Liao, and X. Zhang, "Velocity pointing error analysis for symmetric spinning thrusting Cubesat," Advances in Space Research, vol. 63, no. 11, pp. 3621-3631, 2019.

[10] H. N. Ma, X. Zhang, and W. H. Liao, "Calculation of average power generated by solar array of CubeSat," China Sciencepaper, vol. 13, no. 23, pp. 2700-2703, 2018.

[11] J. Vandersteen, M. Diehl, C. Aerts, and J. Swevers, "Spacecraft attitude estimation and sensor calibration using moving horizon estimation," Journal of Guidance, Control and Dynamics, vol. 36, no. 3, pp. 734-742, 2013.

[12] C. Guo, X. Tong, S. Liu et al., "High-attitude method of sensors and based on filter and Kalman FILTER," ISPRS - International Archives of the Photogrammetry, Remote Sensing and Spatial Information Sciences, vol. XLII-3/W1, pp. 49-53, 2017.

[13] X. B. Yuan, C. Zhang, and C. L. Shi, The cubature Kalman filter and its application in the satellite star-sensor/gyro attitude determination system, China Satellite Navigation Conference, Shanghai, 2017.
[14] D. Lee, G. Vukovich, and R. Lee, "Robust adaptive unscented Kalman filter for spacecraft attitude estimation using quaternion measurements," Journal of Aerospace Engineering, vol. 30, no. 4, pp. 1-11, 2017.

[15] H. A. Hashim, Attitude determination and estimation using vector observations: review, challenges and comparative results, 2020.

[16] H. Ourati and D. E. C. Belkhiat, Multisensor attitude estimation: fundamental concepts and applications, CRC Press, Taylor \& Francis Group, Boca Raton, 2017.

[17] Q. E. U. Rehman, S. Zahir, and N. Abbas, "Attitude estimation for satellites using vector observations," International Research Journal of Engineering and Technology, vol. 6, no. 1, pp. 843849, 2019.

[18] C. Hajiyev and D. C. Guler, "Review on gyroless attitude determination methods for small satellites," Progress in Aerospace Sciences, vol. 90, pp. 54-66, 2017.

[19] B. L. Chen, Y. H. Geng, and X. Yang, High precision attitude estimation algorithm using three star trackers, 10th World Congress on Intelligent Control and Automation, Beijing, 2012.

[20] J. Bae and Y. Kim, Satellite attitude determination and estimation using two star trackers, AIAA Guidance, Navigation, and Control Conference, Toronto, Ontario, Canada, 2010.

[21] D. C. Guler and C. Hajiyev, Error analysis of the vector measurements based attitude determination methods for small satellites, 30th International Symposium on Space Technology and Science, Kobe-Hyogo, Japan, 2015.

[22] M. A. S. I. Mohammed, H. Boussadia, A. Bellar, and A. Adnane, "Performance comparison of attitude determination, attitudeestimation, and nonlinear observers algorithms," Journal of Physics: Conference Series, vol. 783, no. 1, pp. 6274, 2017.

[23] F. L. Markley, Y. Cheng, J. L. Crassidis, and Y. Oshman, "Averaging quaternions," Journal of Guidance, Control and Dynamics, vol. 30, no. 4, pp. 1193-1197, 2007.

[24] F. L. Markley and J. L. Crassidis, Fundamentals of spacecraft attitude determination and control, Space Technology Library, Microcosm Press and Springer, Berlin, 2014.

[25] P. K. Gaurav and S. Kannan, "Star sensor mounting for highly agile earth imaging satellite[J]," Journal of Astrophysics and Astronomy, vol. 41, no. 16, pp. 38-45, 2020.

[26] H. Zhang, H. Zhang, G. Xu, H. Liu, and X. Liu, "Attitude antiinterference federal filtering algorithm for MEMS-SINS/GPS/magnetometer/SV integrated navigation system," Measurement and Control, vol. 53, no. 1-2, pp. 46-60, 2020.

[27] Y. Y. Qin, H. Y. Zhang, and S. H. Wang, Theory of Kalman filter and integrated navigation, Northwestern Polytechnical University Press, Xi'an, 2015.

[28] X. Zhang, X. Zhang, Z. Lu, and W. Liao, "Optimal Path Planning-Based Finite-Time Control for Agile CubeSat Attitude Maneuver," IEEE Access, vol. 7, pp. 102186-102198, 2019. 